A Population Explosion: The Nature and Evolution of X-ray Binaries in Diverse Environments 28 Oct.-2 Nov. 2007, St. Petersburg Beach, FL

R.M. Bandyopadhyay, S. Wachter, D. Gelino, \& C. R. Gelino, eds.

Session: Future Missions and Surveys

\title{
Monitoring LMXBs with the Faulkes Telescopes
}

\author{
Fraser Lewis and Paul Roche \\ LCOGTN, Cardiff University, 5, The Parade, Cardiff, CF24 3AA \\ The Open University, Walton Hall, Milton Keynes, MK' 6 AA \\ David M. Russell and Rob P. Fender \\ University of Amsterdam, Kruislaan 404, 1098 SM Amsterdam \\ University of Southampton, Highfield, Southampton, SO17 1BJ
}

\begin{abstract}
We have been undertaking a monitoring project of 13 low-mass X-ray binaries (LMXBs) using FT North since early 2006. The introduction of FT South has allowed us to extend this monitoring to include 15 southern hemisphere LMXBs (see Figure 1). With new instrumentation, we also intend to expand this monitoring to include both infrared wavelengths and spectroscopy.
\end{abstract}

\section{Introduction}

The Faulkes Telescope Project is an educational and research arm of the Las Cumbres Observatory Global Telescope Network (LCOGTN). It has two 2-metre robotic telescopes, located at Haleakala on Maui (FT North) and Siding Spring in Australia (FT South). It is planned for these telescopes to be complemented by a research network of eighteen 1-metre telescopes located at 6 sites, along with an educational network of twenty-eight 0.4 -metre telescopes, located at 7 sites. This will provide 24 hour coverage of both northern and southern hemispheres. We monitor the brighter systems once per week in $\mathrm{V}, \mathrm{R}$ and i' bands, and the dimmer systems in the i' band. During outbursts or after ATels, we are able to amend this accordingly to increase the observing cadence. Sample results are shown in sections 3 and 4.

\section{Aims}

1. To identify transient outbursts in LMXBs. LMXBs may brighten in the optical/nearinfrared (OIR) for up to a month before X-ray detection. The behaviour of the optical rise is poorly understood, especially for black hole X-ray binaries. Catching outbursts from quiescence will allow us to examine this behaviour and alert the astronomical community to initiate multi-wavelength follow-up observations.

2. To study the variability in quiescence. Recent results have suggested that many processes may contribute to the quiescent optical emission, including emission from the jets in black hole systems, e.g. (Russell et al. 2006). By monitoring the long-term variability of quiescent LMXBs, we aim to provide constraints on the emission processes and the mass functions. 


\begin{tabular}{|c||c|}
\hline FT North & FT South \\
& XTE J0929-314 \\
IGR J00291+5934 & GRS 1009-45 \\
GRO J0422+32 & GRS 1124-68 \\
4U 0614+09 & GS 1354-64 \\
A0620-00 & Cen X-4 \\
XTE J1118+480 & 4U 1543-47 \\
H1705-250 & XTE J1550-564 \\
GRO J1719-24 & 4U 1608-52 \\
XTE J1859+226 & XTE J1650-500 \\
Aq1 X-1 & GRO J1655-40 \\
4U 1957+11 & GX 339-4 \\
GS 2000+25 & XTE J17464-3213 \\
V 404 Cyg & SAX J1808-4-3658 \\
XTE J2123-058 & XTE J1814-338 \\
& HETE J1900.1-2455 \\
\hline
\end{tabular}

Figure 1. List of LMXB Targets

\section{GX339-4}

A target for FT South is the transient black hole binary GX339-4, which went into outburst in early 2007, followed by a steady decline in the following months. The outburst was detected at X-ray (Krimm et al. 2006), OIR (Buxton \& Bailyn 2007) and radio (Corbel et al. 2007) wavelengths. Our observations show that the source continues to decline in both V and i' bands (see Figure 2).

The de-reddened optical spectral energy distribution (SED) during this outburst decay shows a blue spectrum with an excess in the i' band. This excess is weaker in a VLT SED from a low luminosity state in 2005, (Russell and Fender 2008, MNRAS, submitted) as seen in the diagram.

Similar results were obtained from GX339-4 during the decline of the 2002 outburst (Homan et al. 2005), where the excess was attributed to the jet. The results indicate that the OIR contribution from the jet decreases at low luminosities in the hard state, implying that in quiescence the jet will only be detected at lower frequencies, in the mid-infrared. This is consistent with multi-wavelength quiescent observations of a number of LMXBs (Gallo et al. 2007).

\section{Aquila X-1}

Aquila X-1, a transient atoll-type LMXB, went into outburst on May 20, 2007 as observed by INTEGRAL (Rodriguez et al. 2007) and also in the OIR (Maitra \& Bailyn 2007). Since this time, we have been intensively monitoring this target and have observed a more recent burst of activity in V, R and i' bands (Maitra et al. 2007).

Our observations show that in this source at least, the optical and X-ray detections of a new outburst are within a few days of each other (see Figure 3).

\section{Conclusions}

We will continue to monitor these sources in the coming years, complimenting efforts by other teams (e.g. Buxton and Bailyn 2007) and provide long-term light curves that may reveal interesting accretion activity. Already, we detect intrinsic variability in A0620-00, XTE J1118+480, GRO J0422+32 and 4U1957+11. 
Lewis, Russell, Fender and Roche; Monitoring LMXBs with the Faulkes Telescopes

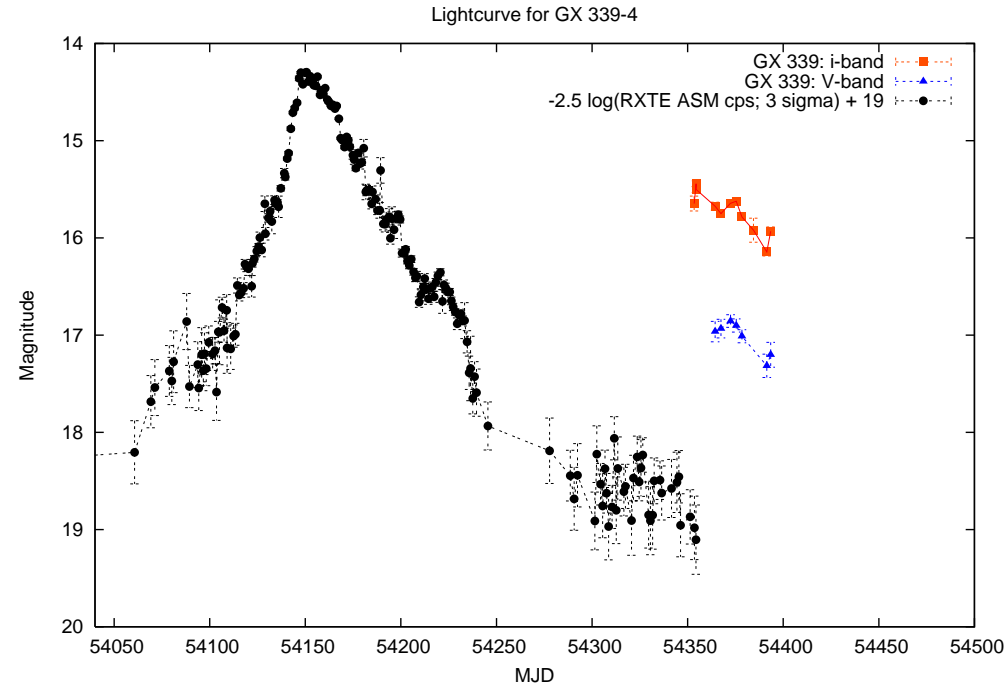

Figure 2. $\quad$ Long-term light curve of GX 339-4

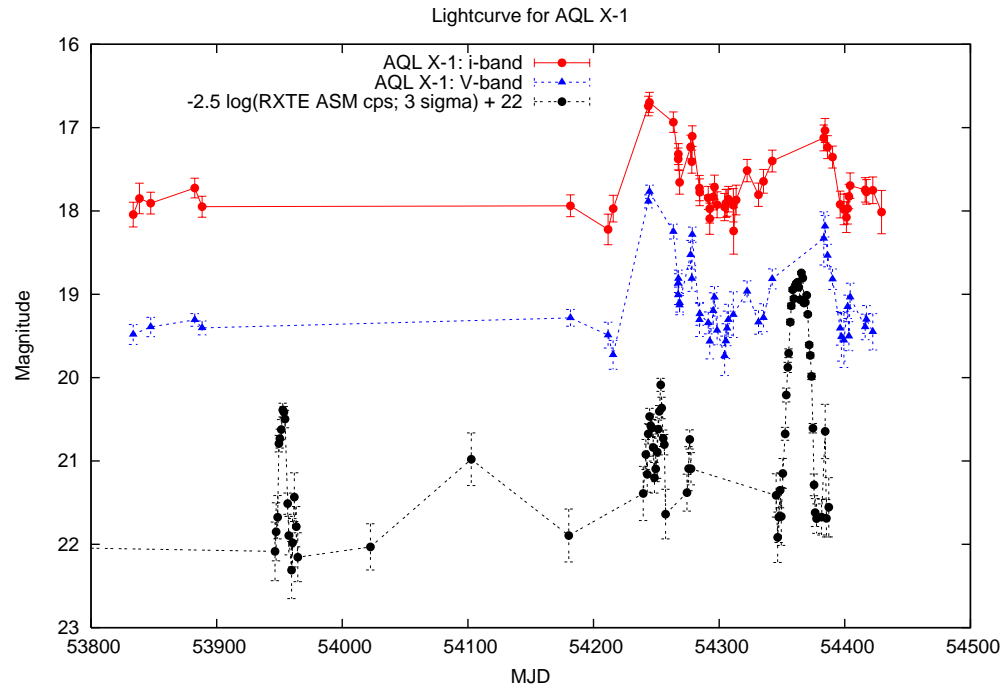

Figure 3. Long-term light curve of Aql X-1

Acknowledgments. FL would like to acknowledge support from the Dill Faulkes Educational Trust.

\section{References}

Buxton \& Bailyn, 2007, ATel, 1027

Corbel et al., 2007, ATel, 1007

Gallo et al., 2007, ApJ, 670, 600

Homan et al., 2005, ApJ, 624, 295

Krimm et al., 2006, ATel, 968

Maitra \& Bailyn, 2007, ATel, 1080

Maitra et al., 2007, ATel, 1218

Rodriguez et al., 2007, ATel, 1079

Russell et al., 2006, MNRAS, 371, 1334 